Document downloaded from:

http://hdl.handle.net/10251/78746

This paper must be cited as:

Blasco De La Cruz, O.; Calabuig, JM.; Sánchez Pérez, EA. (2015). p-Variations of vector measures with respect to vector measures and integral representation of operators. Banach Journal of Mathematical Analysis. 9(1):273-285. doi:10.15352/bjma/09-1-20.

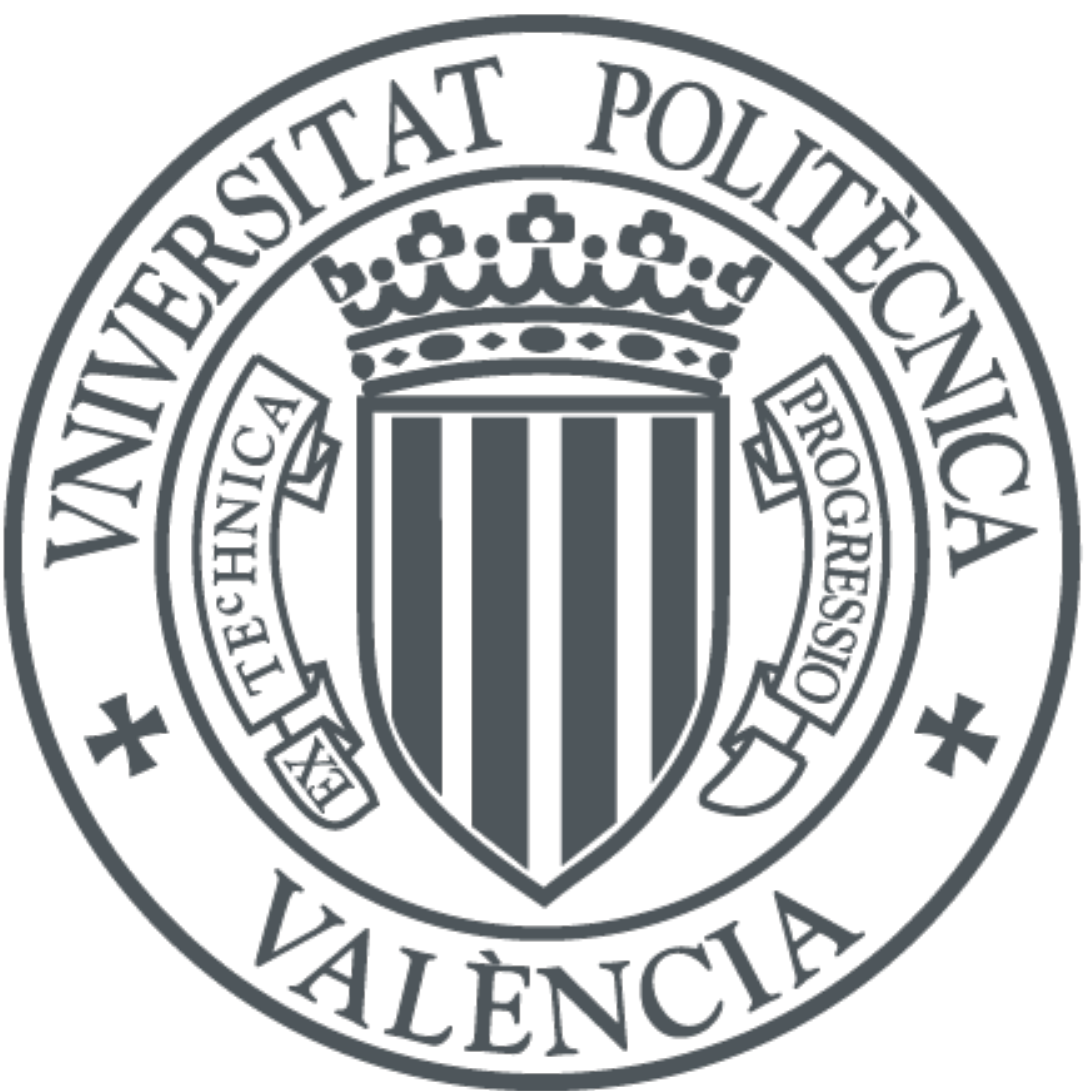

The final publication is available at

http://doi.org/10.15352/bjma/09-1-20

Copyright Duke University Press

Additional Information 


\title{
$p$-VARIATIONS OF VECTOR MEASURES WITH RESPECT TO VECTOR MEASURES AND INTEGRAL REPRESENTATION OF OPERATORS
}

\author{
O. BLASCO ${ }^{1}$, J.M. CALABUIG ${ }^{2 *}$ AND E.A. SÁNCHEZ-PÉREZ ${ }^{3}$
}

\begin{abstract}
In this paper we provide two representation theorems for two relevant classes of operators from any $p$-convex order continuous Banach lattice with weak unit into a Banach space: the class of continuous operators and the class of cone absolutely summing operators. We prove that they can be characterized as spaces of vector measures with finite $p$-semivariation and $p$ variation, respectively, with respect to a fixed vector measure. We give in this way a technique for representing operators as integrals with respect to vector measures.
\end{abstract}

\section{InTRODUCTION}

The theory of integration with respect to a vector measure has its roots in the seminal paper by Bartle, Dunford and Schwartz (see [1]), who developed a Lebesgue type definition for integration of scalar functions with respect to a vector measure in order to extend the Riesz Representation Theorem for the dual space $C(K)^{*}$. More precisely with this integration they represent as integrals weakly compact operators from $C(K)$ spaces into a Banach space $X$. After this paper of the fifties, the interplay between vector measures and operators has been the starting point of a lot of fruitful mathematical developments. Nowadays, a deeper knowledge of the properties of integration of scalar functions with respect to vector measures (see $[11,12,14]$ ) and the corresponding spaces of integrable functions allows a better understanding of the relation between operators on Banach function spaces and their integral representations.

In this paper we show that finite $p$-semivariation and finite $p$-variation of a vector measure with respect to other vector measure characterize some strong relations between the corresponding spaces of integrable functions and also some properties of the integration maps defined between them. When these ideas are written in terms of operators - that is for vector measures defined from operators-, we find that the classes of continuous and cone absolutely summing operators from an order continuous $p$-convex Banach lattice with a weak unit can be represented as integrals of vector measures - as in the case of weakly

Date: Received: xxxxxx; Revised: yyyyyy; Accepted: zzzzzz.

* Corresponding author.

2010 Mathematics Subject Classification. Primary 46E30; Secondary 46G10.

Key words and phrases. Vector measures, operator, $p$-variation, $p$-semivariation, vector valued integration. 
compact operators on $C(K)$ quoted above - having bounded $p$-semivariation or $p$-variation, respectively.

The motivation of our analysis comes from the following well-known result about operators on $L^{p}$-spaces and vector measures that can be already found in the book by Dinculeanu (see [9]). For a finite measure $\mu$, an operator $T$ : $L^{p^{\prime}}(\mu) \rightarrow X$ is continuous if and only if the $p$-semivariation of the vector measure $m_{T}$, denoted by $\left\|m_{T}\right\|_{p}$, and given by

$\left\|m_{T}\right\|_{p}:=\sup \left\{\left(\sum \frac{\left|\left\langle m_{T}\left(A_{i}\right), x^{\prime}\right\rangle\right|^{p}}{\mu\left(A_{i}\right)^{p-1}}\right)^{1 / p}: x^{\prime} \in B_{X^{*}},\left\{A_{i}\right\}\right.$ finite partition of $\left.\Omega\right\}$,

is finite (see for instance [15, Theorem 8] and Example 2.5(1) below for an equivalent formulation). In this paper we prove that this result for operators on $L^{p}$-spaces is in fact a particular example of a general result that holds for $p$-convex Banach lattices involving the $p$-semivariation and the $p$-variation of a vector measure with respect to other vector measure. We provide in this way two vector-measure-type integral representations for continuous and cone absolutely summing operators (Corollary 2.8 and Corollary 3.7, respectively) whose proofs are based on two theorems for vector measures (Theorem 2.6 and 3.5). As the reader may expect, Pettis integrability is involved in the first result (Example 2.5(2)), while Bochner integrability is the key property in the second one (Example 3.4(3)).

Terminology and background. Throughout this paper $X$ and $Y$ denote (real) Banach spaces. By $B_{X}$ we denote the closed unit ball of $X$. Let $L(X, Y)$ be the Banach space of the linear and continuous maps $T$ defined from $X$ into $Y$ endowed with the usual uniform norm $\|T\|=\sup \left\{\|T x\|_{Y}: x \in B_{X}\right\}$. $X^{*}$ will be the topological dual space, that is $X^{*}=L(X, \mathbb{R})$. When $E$ is a Banach lattice an operator $T \in L(E, Y)$ is called cone absolutely summing if there is a constant $k>0$ such that for each finite set of positive elements $x_{1}, \ldots, x_{n} \in E$,

$$
\sum_{i=1}^{n}\left\|T x_{i}\right\| \leq k \sup _{x^{\prime} \in B_{X^{*}}} \sum_{i=1}^{n}\left|\left\langle x_{i}, x^{\prime}\right\rangle\right|=k\left\|\sum_{i=1}^{n} x_{i}\right\|_{X} .
$$

The infimum of such constant is denoted by $\pi_{1}^{+}(T)$. We denote by $\left(\Lambda_{1}^{+}(E, Y), \pi_{1}^{+}\right)$ to the complete space of all cone absolutely summing operators from $E$ into $Y$.

Let $1 \leq p<\infty$. A Banach lattices $E$ is said to be $p$-convex if there exists a constant $k \geq 0$ satisfying that for every finite collection $x_{1}, \ldots, x_{n} \in E$,

$$
\left\|\left(\sum_{i=1}^{n}\left|x_{i}\right|^{p}\right)^{1 / p}\right\| \leq k\left(\sum_{i=1}^{n}\left\|x_{i}\right\|^{p}\right)^{1 / p} .
$$

When every monotone order bounded sequence in the Banach lattice $E$ is convergent (in the norm of $E$ ) then $E$ is said to be order continuous. A weak unit of $E$ is an element $0 \leq e \in E \operatorname{such}$ that $\inf (x, e)=0$ implies $x=0$. The reader is referred to $[8,16]$ to the unexplained terminology and results regarding Banach lattices and absolutely summing operators. 
Let $(\Omega, \Sigma)$ be a measurable space, that is $\Omega$ is a set and $\Sigma$ is a $\sigma$-algebra. For each $A \in \Sigma$ we will denote by $\mathcal{D}_{A}$ the set of all partitions, $\pi_{A}$, of the set $A$ into a finite number of pairwise disjoint elements of $\Sigma$. The characteristic function of the set $A \in \Sigma$ is denoted by $\chi_{A}$. We will also denote by $S_{\Sigma}(X)$ the set of the simple functions, $s=\sum_{i=1}^{n} x_{i} \chi_{A_{i}}$, where $x_{i} \in X$ for all $1 \leq i \leq n$ and $\left\{A_{i}\right\}_{i=1}^{n}$ are pairwise disjoint elements of $\Sigma$.

If $m: \Sigma \rightarrow X$ is a Banach space valued countably additive vector measure, we can define for each functional $x^{\prime} \in X^{*}$ the scalar measure given by $\left\langle m, x^{\prime}\right\rangle(A):=$ $\left\langle m(A), x^{\prime}\right\rangle, A \in \Sigma$. We write $\left|\left\langle m, x^{\prime}\right\rangle\right|$ for its variation. The symbol $\|m\|$ denotes the semivariation of $m$, that is given by $\|m\|(A):=\sup _{x^{\prime} \in B_{X^{*}}}\left|\left\langle m, x^{\prime}\right\rangle\right|(A), A \in \Sigma$. Throughout the paper, $m$ and $\nu$ are countably additive vector measures on the same $\sigma$-algebra $\Sigma$. A set $A \in \Sigma$ is said to be $\|m\|$-null if $\|m\|(A)=0$. We say that $m$ is absolutely continuous with respect to $\nu$, and write $m \ll \nu$, if any measurable set $A$ with $\|\nu\|(A)=0$ satisfies $\|m\|(A)=0$. The reader can find more information on vector measures on our standard reference [7].

A real measurable function $f: \Omega \rightarrow \mathbb{R}$ is integrable with respect to $m$ if for each $x^{\prime} \in X^{*}, f \in L^{1}\left(\left|\left\langle m, x^{\prime}\right\rangle\right|\right)$, and for each $A \in \Sigma$ there is an element $\int_{A} f d m \in X$ such that $\left\langle\int_{A} f d m, x^{\prime}\right\rangle=\int_{A} f d\left\langle m, x^{\prime}\right\rangle$ for all $x^{\prime} \in X^{*}$. If only the first requirement is satisfied, then we say that $f$ is weakly integrable and the corresponding space of such classes of functions (identifying functions that are $\|m\|$-a.e. equal) will by denoted by $L_{w}^{1}(m)$.

Given $1 \leq p<\infty$. The space $L^{p}(m)$ of $p$-integrable function is composed by the equivalence classes of $\|m\|$-a.e. equal integrable functions, with the norm

$$
\|f\|_{L^{p}(m)}:=\sup _{x^{\prime} \in B_{X^{*}}}\left(\int_{\Omega}|f|^{p} d\left|\left\langle m, x^{\prime}\right\rangle\right|\right)^{1 / p}, \quad f \in L^{p}(m) .
$$

For $p=\infty$ we have $L^{\infty}(m)$ the $\|m\|$-essentially bounded measurable functions which it is equipped with the essential supremum norm $\|\cdot\|_{L^{\infty}(m)}$. Considering the $\|m\|$-a.e. order they become Banach lattices, and $L^{p}(m)$ defines, for $1 \leq p<\infty$, an order continuous $p$-convex Banach lattice with weak unit. The space $L_{w}^{p}(m)$ of weakly integrable functions (with the same norm) is also a $p$-convex Banach lattice, and $L^{p}(m) \subseteq L_{w}^{p}(m)$ isometrically. We write $I_{m}$ for the integration map $I_{m}: L^{1}(m) \rightarrow X$ given by $I_{m}(f):=\int_{\Omega} f d m, f \in L^{1}(m)$. It can be easily seen that $L^{p}(m) \subseteq L^{q}(m)$ whenever $1 \leq q \leq p<\infty$. Sometimes we will write $[f]_{m}$ instead of $f$ for an element of $L^{1}(m)$ in order to recall that $f$ is in fact an equivalence class of functions. The reader can find further information on these spaces in [14, Ch.3].

We denote $L^{0}(m)$ the space of equivalence classes $[f]_{m}$ of measurable functions. Hence if we assume that $m \ll \nu$ then the map id : $L^{0}(\nu) \rightarrow L^{0}(m)$ given by $\operatorname{id}\left([f]_{\nu}\right)=[f]_{m}$ is well-defined. For $1 \leq q \leq p \leq \infty$ we introduce the symbol $L^{p}(\nu) \Subset L^{q}(m)$ to mean that id $: L^{p}(\nu) \rightarrow L^{q}(m)$ is well-defined. Notice that, since this map is positive, it is always continuous (see [13, p.2]). Although this relation is not strictly speaking an inclusion, we use the symbol "id" to denote it. For the particular case that we deal with in the present paper — vector measures defined by operators - the reader can find a complete explanation of this kind of relation in [6, Section 3]. 
Finally if $1 \leq p \leq \infty$ as usual we write $p^{\prime} \in[1, \infty]$ the (extended) real number given by $1 / p+1 / p^{\prime}=1$.

\section{MEASURES OF BOUNDED $p$-SEMIVARIATION.}

Let us start by expressing the absolute continuity of $m$ with respect to $\nu$ in terms of inclusion between spaces.

Proposition 2.1. Let $m: \Sigma \rightarrow X$ and $\nu: \Sigma \rightarrow Y$ be countable additive vector measures. The following assertions are equivalent:

(1) $m \ll \nu$.

(2) $L^{\infty}(\nu) \Subset L^{\infty}(m)$.

(3) $L^{\infty}(\nu) \Subset L^{1}(m)$.

(4) The integration map $I_{m}^{\infty}: L^{\infty}(\nu) \rightarrow X$ is continuous.

Proof. $(1) \Rightarrow(2)$. Clearly for a fixed $f \in L^{\infty}(\nu)$ the measurable set

$$
A=\left\{w \in \Omega:|f(w)|>\|f\|_{L^{\infty}(\nu)}\right\}
$$

is $\|\nu\|$-null so by using (1) it is also $\|m\|$-null and $\|f\|_{L^{\infty}(m)} \leq\|f\|_{L^{\infty}(\nu)}$. This gives $(2)$. $(2) \Rightarrow(3)$ follows from the continuous inclusion $L^{\infty}(m) \Subset L^{1}(m)$. The implication $(3) \Rightarrow(4)$ is a direct consequence of the continuity of the integration map $I_{m}^{1}: L^{1}(m) \rightarrow X$. Finally let us see that $(4) \Rightarrow(1)$. Take $A \in \Sigma$ such that $\|\nu\|(A)=0$. Then $I_{m}^{\infty}\left(\chi_{B}\right)=m(B)=0$ for any $B \in \Sigma$ and $B \subseteq A$. Therefore $\|m\|(A)=0$.

It is easy to give examples of couples of measures $(m, \nu)$ such that $m \ll \nu$. Let us introduce some of them which will appear in the sequel.

Example 2.2. (1) If $\nu: \Sigma \rightarrow Y$ is a vector measure and $h \in L^{1}(\nu)$ let us consider the countable additive vector measure $\nu_{h}: \Sigma \rightarrow Y$ given by

$$
\nu_{h}(A)=\int_{A} h d \nu, \quad A \in \Sigma .
$$

It is clear that $\nu_{h} \ll \nu$.

(2) Consider now a vector measure $\nu: \Sigma \rightarrow X$ and take $T \in L(X, Y)$. Then for the new vector measure $T \circ \nu: \Sigma \rightarrow Y$ given by $T \circ \nu(A)=T(\nu(A))$, for all $A \in \Sigma$, it is clear that $T \circ \nu \ll \nu$.

(3) Let $(\Omega, \Sigma, \mu)$ be a finite measure space, $1 \leq q<\infty$ and denote by $m_{q}: \Sigma \rightarrow$ $L^{q}(\mu)$ the vector measure given by $m_{q}(A)=\chi_{A}$ for all $A \in \Sigma$ then $m_{q} \ll \mu$.

(4) Finally let $\nu: \Sigma \rightarrow X$ be a vector measure and $T \in L\left(L^{\infty}(\nu), X\right)$. Denote by $m_{T}: \Sigma \rightarrow X$ the set function given by $m_{T}(A)=T\left(\chi_{A}\right)$, for all $A \in \Sigma$. In the case when $m_{T}$ is countable additive - for instance if $T$ is order continuoustherefore it is easy to see that $m_{T} \ll \nu$.

Definition 2.3. Let $1<p \leq \infty$ and let $m: \Sigma \rightarrow X$ and $\nu: \Sigma \rightarrow Y$ be countable additive vector measures. We say that $m$ has bounded $p$-semivariation with respect to $\nu$ if

$$
\|m\|_{p, \nu}=\sup \left\{\left\|\sum_{A \in \pi} \alpha_{A} m(A)\right\|_{X}: \pi \in \mathcal{D}_{\Omega}, \sum_{A \in \pi} \alpha_{A} \chi_{A} \in B_{L^{p^{\prime}}(\nu)}\right\}<\infty .
$$


For the effective computation of $\|\cdot\|_{p, \nu}$, notice that for every $\pi \in \mathcal{D}_{\Omega}$,

$$
\sum_{A \in \pi} \alpha_{A} \chi_{A} \in B_{L^{p^{\prime}}(\nu)} \Longleftrightarrow \sum_{A \in \pi} \alpha_{A} \chi_{A} \in B_{L^{p^{\prime}}\left(\left|\left\langle\nu, y^{\prime}\right\rangle\right|\right)} \text {, for all } y^{\prime} \in B_{Y^{*}}
$$

Note also that finite $p$-semivariation with respect to $\nu$ of $m$ implies in particular absolute continuity of $m$ with respect to $\nu$, i.e. $\|\nu\|(A)=0$ implies that $\|m\|(A)=$ 0 for each $A \in \Sigma$.

Of course in the case $\nu=\mu$ where $(\Omega, \Sigma, \mu)$ is a positive finite measure space and $1<p<\infty$ the classical notion of $p$-semivariation of a vector valued measure $m: \Sigma \rightarrow X$, that is to say

$$
\|m\|_{p}=\sup \left\{\left(\sum_{A \in \pi} \frac{\left|\left\langle m(A), x^{\prime}\right\rangle\right|^{p}}{\mu(A)^{p-1}}\right)^{1 / p}: x^{\prime} \in B_{X^{*}}, \pi \in \mathcal{D}_{\Omega}\right\},
$$

corresponds to $\|m\|_{p}=\|m\|_{p, \mu}$. However we see in the next example that also coincides with the $q$-variation with respect to different vector-valued measures.

Proposition 2.4. Let $(\Omega, \Sigma, \mu)$ be a positive finite measure space, $m: \Sigma \rightarrow X$ be a vector valued measure and $1<p \leq r<\infty$. Then there exist a Banach space $Y$ and a vector valued measure $\nu: \Sigma \rightarrow Y$ such that $\|m\|_{p}=\|m\|_{r, \nu}$.

Proof. Let $Y=L^{q}(\mu)$ for $q=p^{\prime} / r^{\prime} \geq 1$ and let $\nu$ be the measure

$$
\nu=m_{q}: \Sigma \rightarrow L^{q}(\mu), \quad m_{q}(A)=\chi_{A} .
$$

Using that $L^{q}(\mu)=L^{1}(\nu)$ one has that for every $\pi \in \mathcal{D}_{\Omega}$

$$
\left\|\sum_{A \in \pi} \alpha_{A} \chi_{A}\right\|_{L^{r^{\prime}\left(m_{q}\right)}}=\left\|\sum_{A \in \pi} \alpha_{A} \chi_{A}\right\|_{L^{p^{\prime}}(\mu)} .
$$

Therefore

$$
\|m\|_{r, \nu}=\sup \left\{\left\|\sum_{A \in \pi} \alpha_{A} m(A)\right\|_{X}: \pi \in \mathcal{D}_{\Omega}, \sum_{A \in \pi} \alpha_{A} \chi_{A} \in B_{L^{r^{\prime}\left(m_{q}\right)}}\right\}=\|m\|_{p} .
$$

Example 2.5. (1) Let $T: L^{p^{\prime}}(\mu) \rightarrow X$ be a bounded linear map and $m_{T}: \Sigma \rightarrow$ $X$ the vector measure associated to $T$, given by $m_{T}(A)=T\left(\chi_{A}\right)$. Then $m_{T}$ has bounded $p$-semivariation with respect to $\mu$ and $\left\|m_{T}\right\|_{p, \mu}=\|T\|$. Moreover, in particular, $\left\|m_{q}\right\|_{p}=1$ for $q \leq p^{\prime}$.

Indeed, the first part is clear since

$$
\begin{aligned}
\left\|m_{T}\right\|_{p, \mu} & =\sup \left\{\left\|\sum_{A \in \pi} \alpha_{A} m_{T}(A)\right\|_{X}: \pi \in \mathcal{D}_{\Omega}, \sum_{A \in \pi} \alpha_{A} \chi_{A} \in B_{L^{p^{\prime}}(\mu)}\right\} \\
& =\sup \left\{\left\|T\left(\sum_{A \in \pi} \alpha_{A} \chi_{A}\right)\right\|_{X}: \pi \in \mathcal{D}_{\Omega}, \sum_{A \in \pi} \alpha_{A} \chi_{A} \in B_{L^{p^{\prime}}(\mu)}\right\} \\
& =\|T\| .
\end{aligned}
$$


In particular, for $q \leq p^{\prime}$, the inclusion map $T=\mathrm{id}: L^{p^{\prime}}(\mu) \rightarrow L^{q}(\mu)$ defines the vector measure $m_{q}=m_{\mathrm{id}}: \Sigma \rightarrow L^{q}(\mu)$ given by $m_{q}(A)=\chi_{A}$ which has bounded $p$-semivariation with respect to $\mu$ and $\left\|m_{q}\right\|_{p}=\left\|m_{q}\right\|_{p, \mu}=\|\operatorname{id}\|_{L^{p^{\prime}}(\mu) \rightarrow L^{q}(\mu)}=1$.

(2) Let $\mathbf{f}: \Omega \rightarrow X$ be a Pettis $p$-integrable function and $m_{\mathbf{f}}: \Sigma \rightarrow X$ the vector measure $m_{\mathbf{f}}: \Sigma \rightarrow X$ defined by $m_{\mathbf{f}}(A)=\int_{A} \mathbf{f} d \mu$, where the integral stands for the Pettis integral of $\mathbf{f}$. Then $m_{\mathbf{f}}$ has bounded $p$-semivariation with respect to $\mu$ and $\left\|m_{\mathbf{f}}\right\|_{p, \mu}=\|\mathbf{f}\|_{P_{p}(X)}$-where $P_{p}(X)$ is the space consisting of Pettis $p$-integrable functions - Indeed, just consider the measure $m_{\mathbf{f}}=m_{T_{\mathbf{f}}}$ where

$$
T_{\mathbf{f}}(\phi)=\int_{\Omega} \phi \mathbf{f} d \mu, \quad \phi \in L^{p^{\prime}}(\mu)
$$

and recall that $\left\|T_{\mathbf{f}}\right\|=\|\mathbf{f}\|_{P_{p}(X)}$.

In our first important result we characterize when a vector measure $m: \Sigma \rightarrow X$ has bounded $p$-semivariation with respect to other vector measure $\nu: \Sigma \rightarrow Y$.

Theorem 2.6. Let $1<p \leq \infty$ and let $m: \Sigma \rightarrow X$ and $\nu: \Sigma \rightarrow Y$ be countable additive vector measures. The following assertions are equivalent:

(1) $\|m\|_{p, \nu}<\infty$.

(2) There exists a bounded linear map $T_{m}: L^{p^{\prime}}(\nu) \rightarrow X$ such that $T_{m}\left(\chi_{A}\right)=$ $m(A)$, for all $A \in \Sigma$.

(3) $L^{p^{\prime}}(\nu) \Subset L^{1}(m)$.

(4) The integration map $I_{m}^{p^{\prime}}: L^{p^{\prime}}(\nu) \rightarrow X$ is continuous.

Moreover, $\|m\|_{p, \nu}=\left\|T_{m}\right\|=\|\mathrm{id}\|_{L^{p^{\prime}(\nu) \rightarrow L^{1}(m)}}=\left\|I_{m}^{p^{\prime}}\right\|_{L^{p^{\prime}}(\nu) \rightarrow X}$.

Proof. For $(1) \Rightarrow(2)$ let us consider a simple function $s=\sum_{j=1}^{n} \alpha_{j} \chi_{A_{j}}$ and define the bounded linear map $T_{m}(s)=\sum_{j=1}^{n} \alpha_{j} m\left(A_{j}\right) \in X$. Since

$$
\left\|T_{m}(s)\right\|_{X}=\left\|\sum_{j=1}^{n} \alpha_{j} m\left(A_{j}\right)\right\|_{X} \leq\|m\|_{p, \nu}\|s\|_{L^{p^{\prime}(\nu)}},
$$

by the density of simple functions in $L^{p^{\prime}}(\nu)$ we can extend $T_{m}: L^{p^{\prime}}(\nu) \rightarrow X$ with

$$
\left\|T_{m}\right\| \leq\|m\|_{p, \nu}
$$

In order to prove that $(2) \Rightarrow(3)$ first observe that the vector measure associated to $T_{m}, m_{T_{m}}$, is exactly the measure $m$. This means that $L^{1}\left(m_{T_{m}}\right)=L^{1}(m)$. On the other hand, $L^{p^{\prime}}(\nu)$ is a Banach function space over a Rybakov measure for $\nu$ as $\lambda=\left|\left\langle\nu, y_{0}^{\prime}\right\rangle\right|, y_{0}^{\prime} \in Y^{*}$. Hence by the Optimal Domain Theorem (see [14, Theorem 4.14] and the more general factorization version given in Section 3 of [6]) there is an inclusion/quotient map from the space $L^{p^{\prime}}(\nu)$ to $L^{1}\left(m_{T_{m}}\right)=L^{1}(m)$, i.e $L^{p^{\prime}}(\nu) \Subset L^{1}(m)$.

Let us show that $(3) \Rightarrow(4)$. Take $f \in L^{p^{\prime}}(\nu)$,

$$
\left\|I_{m}^{p^{\prime}}(f)\right\|_{X}=\left\|\int_{\Omega} f d m\right\|_{X} \leq\|f\|_{L^{1}(m)} \leq\|\mathrm{id}\|_{L^{p^{\prime}(\nu) \rightarrow L^{1}(m)}}\|f\|_{L^{p^{\prime}(\nu)}} .
$$

Therefore $I_{m}^{p^{\prime}}$ is continuous and

$$
\left\|I_{m}^{p^{\prime}}\right\|_{L^{p^{\prime}}(\nu) \rightarrow X} \leq\|\mathrm{id}\|_{L^{p^{\prime}(\nu) \rightarrow L^{1}(m)}} .
$$


We prove now $(4) \Rightarrow(1)$. Take $\pi \in D_{\Omega}$,

$$
\begin{aligned}
\left\|\sum_{A \in \pi} \alpha_{A} m(A)\right\|_{X} & =\left\|\int_{\Omega}\left(\sum_{A \in \pi} \alpha_{A} \chi_{A}\right) d m\right\|_{X}=\left\|I_{m}^{p^{\prime}}\left(\sum_{A \in \pi} \alpha_{A} \chi_{A}\right)\right\|_{X} \\
& \leq\left\|I_{m}^{p^{\prime}}\right\|_{L^{p^{\prime}}(\nu) \rightarrow X}\left\|\sum_{A \in \pi} \alpha_{A} \chi_{A}\right\|_{L^{p^{\prime}}(\nu)} .
\end{aligned}
$$

Therefore $\|m\|_{p, \nu}$ is finite and

$$
\|m\|_{p, \nu} \leq\left\|I_{m}^{p^{\prime}}\right\|_{L^{p^{\prime}(\nu) \rightarrow X}} .
$$

From the inequalities (2.1), (2.2) and (2.3) in order to finish the proof we have to show that $\|\mathrm{id}\|_{L^{p^{\prime}(\nu) \rightarrow L^{1}(m)}} \leq\left\|T_{m}\right\|$. Take $f \in L^{p^{\prime}}(\nu)$,

$$
\begin{aligned}
\|f\|_{L^{1}(m)} & =\sup \left\{\left\|\int_{\Omega} s f d m\right\|_{X}: s \in S_{\Sigma}(\mathbb{R}) \cap B_{L^{\infty}(\nu)}\right\} \\
& =\sup \left\{\left\|T_{m}(s f)\right\|_{X}: s \in S_{\Sigma}(\mathbb{R}) \cap B_{L^{\infty}(\nu)}\right\} \\
& \leq \sup \left\{\left\|T_{m}\right\| \cdot\|s f\|_{L^{p^{\prime}(\nu)}}: s \in S_{\Sigma}(\mathbb{R}) \cap B_{L^{\infty}(\nu)}\right\} \\
& \leq\left\|T_{m}\right\| \cdot\|f\|_{L^{p^{\prime}}(\nu)} .
\end{aligned}
$$

Hence $\|\mathrm{id}\|_{L^{p^{\prime}(\nu) \rightarrow L^{1}(m)}} \leq\left\|T_{m}\right\|$.

The vector space consisting of those measures $m$ appearing in Theorem 2.6 is denoted by $\mathcal{V}_{X}^{p}(\nu)$. The previous result allows us to establish an isometric isomorphism between $\mathcal{V}_{X}^{p}(\nu)$ and $L\left(L^{p^{\prime}}(\nu), X\right)$. Therefore,

Corollary 2.7. Let $1<p \leq \infty, \nu: \Sigma \rightarrow Y$ a countable additive vector measure and $X$ a Banach space. Then $\mathcal{V}_{X}^{p}(\nu)$ is a Banach space under the norm $\|\cdot\|_{p, \nu}$ that can be identified isometrically with $L\left(L^{p^{\prime}}(\nu), X\right)$.

Theorem 2.6 is in fact an integral representation theorem for operators from a $p^{\prime}$-convex order continuous Banach lattice on a Banach space. This can be proved by using some well-known results on representations of this class of Banach lattices. Recall that other implicit fact that is assumed is that $\nu$ and $m$ must be defined on the same measure space $(\Omega, \Sigma)$.

Corollary 2.8. Let $1<p \leq \infty$. Let $E$ be an order continuous $p^{\prime}$-convex Banach lattice with a weak unit and $X$ a Banach space. Then there is a measure space $(\Omega, \Sigma)$ and a vector measure $\nu$ on it such that $\mathcal{V}_{X}^{p}(\nu)=L(E, X)$ (isomorphically). In particular, there is an order isomorphism $I: E \rightarrow L^{p^{\prime}}(\nu)$ satisfying that for each $T \in L(E, X)$ there is a vector measure $m_{T} \ll \nu$ such that $\left\|m_{T}\right\|_{p, \nu}<\infty$ and $T(\cdot)=\int I(\cdot) d m_{T}$.

Proof. This is a consequence of the representation theorem for Banach lattices using vector measures: if $E$ satisfies the requirements above, there is a vector measure $\nu$ such that there is an order isomorphism between $E$ and $L^{p^{\prime}}(\nu)$ (see [14, Prop.3.30] and the references therein). Therefore, each $T \in L(E, X)$ defines an operator $S_{T}: L^{p^{\prime}}(\nu) \rightarrow X$. For getting the integral formula, it is enough to use Theorem 2.6 with the vector measure $m_{T}$ playing the role of $m$ in (2). 
Notice that the integral representation obtained above can be written for each vector measure representing the Banach lattice $E$. For instance, Lebesgue measure $\mu$ on $[0,1]$ can be obviously used for representing the space $L^{p^{\prime}}[0,1]$ as $L^{p^{\prime}}(\mu)$. This is Example 2.5(1). The other cases in it show more classical examples when we deal with the $p$-semivariation of vector valued measures with respect to scalar ones. Now we present a different example where both measures are vector valued.

Corollary 2.9. Let $1<p \leq \infty, \nu: \Sigma \rightarrow Y$ a countable additive vector measure and $h \in L^{1}(\nu)$. The following assertions are equivalent:

(1) $\left\|\nu_{h}\right\|_{p, \nu}<\infty$.

(2) $L^{p^{\prime}}(\nu) \Subset L^{1}\left(\nu_{h}\right)$.

(3) $h \in L^{p}(\nu)$.

Moreover, $\left\|\nu_{h}\right\|_{p, \nu}=\|h\|_{L^{p}(\nu)}=\|\mathrm{id}\|_{L^{p^{\prime}(\nu) \rightarrow L^{1}\left(\nu_{h}\right)}}$.

Proof. Theorem 2.6 gives that $(1) \Rightarrow(2)$ and

$$
\left\|\nu_{h}\right\|_{p, \nu}=\|\mathrm{id}\|_{L^{p^{\prime}}(\nu) \rightarrow L^{1}\left(\nu_{h}\right)} .
$$

Let us proof $(2) \Rightarrow(3)$. Recall that the space $M\left(L_{w}^{p^{\prime}}(\nu), L^{1}(\nu)\right)$ of multiplication operators from $L_{w}^{p^{\prime}}(\nu)$ into $L^{1}(\nu)$ endowed with the operator norm is isometrically isomorphic to $L^{p}(\nu)$ with the norm $\|\cdot\|_{L^{p}(\nu)}$ (cf. [14, Proposition 3.43]). Take $g \in L_{w}^{p^{\prime}}(\nu)$ and use (2) to obtain

$$
\|h g\|_{L^{1}(\nu)}=\|g\|_{L^{1}\left(\nu_{h}\right)} \leq\|\mathrm{id}\|_{L^{p^{\prime}(\nu) \rightarrow L^{1}\left(\nu_{h}\right)}}\|g\|_{L^{p^{\prime}(\nu)}} .
$$

Hence $h \in M\left(L_{w}^{p^{\prime}}(\nu), L^{1}(\nu)\right)=L^{p}(\nu)$ and

$$
\|h\|_{L^{p}(\nu)} \leq\|\mathrm{id}\|_{L^{p^{\prime}(\nu) \rightarrow L^{1}\left(\nu_{h}\right)}} .
$$

We show now that $(3) \Rightarrow(1)$. Take $\pi \in \mathcal{D}_{\Omega}$,

$$
\begin{aligned}
\left\|\sum_{A \in \pi} \alpha_{A} \nu_{h}(A)\right\|_{Y} & =\left\|\int_{\Omega}\left(\sum_{A \in \pi} \alpha_{A} \chi_{A}\right) h d \nu\right\|_{Y} \leq\left\|\left(\sum_{A \in \pi} \alpha_{A} \chi_{A}\right) h\right\|_{L^{1}(\nu)} \\
& \leq\left\|\sum_{A \in \pi} \alpha_{A} \chi_{A}\right\|_{L^{p^{\prime}(\nu)}}\|h\|_{L^{p}(\nu)} .
\end{aligned}
$$

Therefore we obtain that

$$
\left\|\nu_{h}\right\|_{p, \nu} \leq\|h\|_{L^{p}(\nu)} \text {. }
$$

Finally using (2.5), the last part of Theorem 2.6 and (2.6) we have

$$
\|h\|_{L^{p}(\nu)} \leq\|\mathrm{id}\|_{L^{p^{\prime}(\nu) \rightarrow L^{1}\left(\nu_{h}\right)}}=\left\|\nu_{h}\right\|_{p, \nu} \leq\|h\|_{L^{p}(\nu)} .
$$

The last result of this section is other consequence of Theorem 2.6 and provides a new characterization of the norm of the spaces $L^{p}(\nu)$ in our setting.

Corollary 2.10. Let $1<p<\infty, \nu: \Sigma \rightarrow Y$ a countable additive vector measure and $h \in L^{p}(\nu)$. Then

$$
\|h\|_{L^{p}(\nu)}=\sup \left\{\|h\|_{L^{1}(m)}: m \in B_{\mathcal{V}_{Y}^{p^{\prime}}(\nu)}\right\} .
$$


Proof. For the first inequality observe that since $h \in L^{p}(\nu)$ then $h^{p-1} \in L^{p^{\prime}}(\nu)$. By using Corollary 2.9 with this function $h^{p-1}$ and the space $L^{p^{\prime}}(\nu)$ we have that

$$
\left\|\nu_{h^{p-1}}\right\|_{p^{\prime}, \nu}=\left\||h|^{p-1}\right\|_{L^{p^{\prime}(\nu)}}=\|h\|_{L^{p}(\nu)}^{p-1} .
$$

Consider now the vector valued measure $m=\|h\|_{L^{p}(\nu)}^{1-p} \nu_{h^{p-1}}=\nu_{\|h\|_{L^{p}(\nu)}^{1-p} h^{p-1}}$ that satisfies $\|m\|_{p^{\prime}, \nu}=1$ and

$$
\|h\|_{L^{1}(m)}=\||h| \cdot\| h\left\|_{L^{p}(\nu)}^{1-p}|h|^{p-1}\right\|_{L^{1}(\nu)}=\|h\|_{L^{p}(\nu)}^{1-p}\left\||h|^{p}\right\|_{L^{1}(\nu)}=\|h\|_{L^{p}(\nu)} .
$$

This gives

$$
\|h\|_{L^{p}(\nu)} \leq \sup \left\{\|h\|_{L^{1}(m)}: m \in B_{\nu_{Y}^{p^{\prime}(\nu)}}\right\} .
$$

For the other inequality take $m \in B_{\mathcal{V}_{Y}^{p^{\prime}}(\nu)}$. Using Theorem 2.6 we have that $L^{p}(\nu) \Subset L^{1}(m)$. Since moreover $\|\mathrm{id}\|_{L^{p}(\nu) \rightarrow L^{1}(m)}=\|m\|_{p^{\prime}, \nu} \leq 1$ we obtain

$$
\|h\|_{L^{1}(m)} \leq\|\operatorname{id}\|_{L^{p}(\nu) \rightarrow L^{1}(m)}\|h\|_{L^{p}(\nu)} \leq\|h\|_{L^{p}(\nu)} .
$$

Since $m \in B_{\mathcal{V}_{Y}^{p^{\prime}}(\nu)}$ is arbitrary we have the other inequality and hence the equality.

\section{MeAsures of BOUNDED $p$-VARIATION.}

In this section we develop the counterpart of the results of the previous one for the case of finite $p$-variation instead of finite $p$-semivariation. As we will show, this leads us to represent the class of cone absolutely summming operators.

Definition 3.1. Let $1<p \leq \infty$ and let $m: \Sigma \rightarrow X$ and $\nu: \Sigma \rightarrow Y$ be countable additive vector measures. We say that $m$ has bounded $p$-variation with respect to $\nu$ if

$$
|m|_{p, \nu}=\sup \left\{\sum_{A \in \pi}\left|\alpha_{A}\right|\|m(A)\|_{X}: \pi \in \mathcal{D}_{\Omega}, \sum_{A \in \pi} \alpha_{A} \chi_{A} \in B_{L^{p^{\prime}}(\nu)}\right\}<\infty .
$$

Remark 3.2. If $m$ has bounded $p$-variation with respect to $\nu$ then given $A \in \Sigma$, $\pi \in \mathcal{D}_{A}$,

$$
\sum_{B \in \pi}\|m(B)\|_{X} \leq|m|_{p, \nu}\left\|\sum_{B \in \pi} \chi_{B}\right\|_{L^{p^{\prime}(\nu)}} \leq|m|_{p, \nu}\|\nu\|(A)^{1 / p^{\prime}} .
$$

In particular, $m$ has bounded variation $|m|$ and $|m| \ll \nu$.

As in the previous section we present now some easy examples. Of course in the case $\nu=\mu$ where $(\Omega, \Sigma, \mu)$ is a positive finite measure space and $1<p<\infty$ the classical notion of $p$-variation of a vector valued measure $m: \Sigma \rightarrow X$, that is to say

$$
|m|_{p}=\sup \left\{\left(\sum_{A \in \pi} \frac{\|m(A)\|^{p}}{\mu(A)^{p-1}}\right)^{1 / p}: \pi \in \mathcal{D}_{\Omega}\right\}
$$

corresponds to $|m|_{p}=|m|_{p, \mu}$. Similarly as in Proposition 2.4 we have the analogue result. 
Proposition 3.3. Let $(\Omega, \Sigma, \mu)$ be a positive finite measure space, $m: \Sigma \rightarrow X$ be a vector valued measure and $1<p \leq r<\infty$. Then there exist a Banach space $Y$ and a vector valued measure $\nu: \Sigma \rightarrow Y$ such that $|m|_{p}=|m|_{r, \nu}$.

Example 3.4. Let $(\Omega, \Sigma, \mu)$ be a positive finite measure space and $1<p<\infty$. (1) If $T: L^{p^{\prime}}(\mu) \rightarrow X$ is a cone absolutely summing operator then $m_{T}$ has bounded $p$-variation with respect to $\mu$ and $\left|m_{T}\right|_{p, \mu}=\pi_{1}^{+}(T)$.

(2) Let $\mathbf{f}: \Omega \rightarrow X$ be a Bochner $p$-integrable function and consider $m_{\mathbf{f}}: \Sigma \rightarrow$ $X$ defined by $m_{\mathbf{f}}(A)=\int_{A} \mathbf{f} d \mu$, where now the integral stands for the Bochner integral of $\mathbf{f}$. Then $m_{\mathbf{f}}$ has bounded $p$-variation with respect to $\mu$ and

$$
\left|m_{\mathbf{f}}\right|_{p, \mu}=\left|m_{\mathbf{f}}\right|_{p}=\left(\int_{\Omega}\|\mathbf{f}\|^{p} d \mu\right)^{\frac{1}{p}} .
$$

We prove now the main result of this section (similar results can also be found in [2], [3], [4], [5] and [10]).

Theorem 3.5. Let $1<p \leq \infty$ and let $m: \Sigma \rightarrow X$ and $\nu: \Sigma \rightarrow Y$ be countable additive vector measures. The following assertions are equivalent:

(1) $|m|_{p, \nu}<\infty$.

(2) $L^{p^{\prime}}(\nu) \Subset L^{1}(|m|)$.

(3) The integration map $I_{m}^{p^{\prime}}: L^{p^{\prime}}(\nu) \rightarrow X$ is cone absolutely summing.

(4) There exists a cone absolutely summing operator $T_{m}: L^{p^{\prime}}(\nu) \rightarrow X$ such that $T_{m}\left(\chi_{A}\right)=m(A)$, for all $A \in \Sigma$.

Moreover, $|m|_{p, \nu}=\|\mathrm{id}\|_{L^{p^{\prime}(\nu) \rightarrow L^{1}(|m|)}}=\pi_{1}^{+}\left(\left.I_{m}^{p^{\prime}}\right|_{L^{p^{\prime}(\nu)}}\right)=\left\|T_{m}\right\|$.

Proof. Let us start by proving $(1) \Rightarrow(2)$. Take $\pi=\left\{A_{i}\right\}_{i=1}^{n} \in \mathcal{D}_{\Omega}$ and we will show that if $s=\sum_{i=1}^{n} \alpha_{A_{i}} \chi_{A_{i}}$ is a simple function then

$$
\|s\|_{L^{1}(|m|)} \leq|m|_{p, \nu}\|s\|_{L^{p^{\prime}(\nu)}} .
$$

Let $\varepsilon>0$. For each $1 \leq i \leq n$ take a partition $\pi_{i}=\left\{B_{j}^{i}\right\}_{j=1}^{k(i)}$ of $A_{i} \in \pi$ such that

$$
\sum_{B_{j}^{i} \in \pi_{i}}\left\|m\left(B_{j}^{i}\right)\right\|>|m|\left(A_{i}\right)-\frac{\varepsilon}{2^{i}\left|\alpha_{A_{i}}\right|} .
$$

Hence, by using (1)

$$
\begin{aligned}
\sum_{i=1}^{n}\left|\alpha_{A_{i}}\right| \cdot|m|\left(A_{i}\right) & \leq \sum_{i=1}^{n} \sum_{B_{j}^{i} \in \pi_{i}}\left|\alpha_{A_{i}}\right|\left\|m\left(B_{j}^{i}\right)\right\|_{X}+\varepsilon \\
& \leq|m|_{p, \nu}\left\|\sum_{i=1}^{n} \sum_{B_{j}^{i} \in \pi_{i}} \alpha_{A_{i}} \chi_{B_{j}^{i}}\right\|_{L^{p^{\prime}(\nu)}}+\varepsilon \\
& \leq|m|_{p, \nu}\left\|\sum_{i=1}^{n} \alpha_{A_{i}} \chi_{A_{i}}\right\|_{L^{p^{\prime}(\nu)}}+\varepsilon .
\end{aligned}
$$


Taking the limit as $\varepsilon$ goes to zero one gets (3.1). The density of the simple functions both in $L^{1}(|m|)$ and $L^{p^{\prime}}(\nu)$ gives

$$
\|\mathrm{id}\|_{L^{p^{\prime}(\nu) \rightarrow L^{1}(|m|)}} \leq|m|_{p, \nu} .
$$

Let us continue with the proof of $(2) \Rightarrow(3)$. Recall that for a non-negative finite measure any bounded operator $T: L^{1}(\mu) \rightarrow X$ is cone absolutely summing with $\pi_{1}^{+}(T)=\|T\|$ (cf. [2]). Now using that the composition of a positive operator and a cone absolutely summing operator is cone absolutely summing (3) follows from (2) because $I_{m}^{p^{\prime}}$ is obviously bounded from $L^{1}(|m|)$ to $X$. Moreover

$$
\pi_{1}^{+}\left(\left.I_{m}^{p^{\prime}}\right|_{L^{p^{\prime}(\nu)}}\right) \leq\|\mathrm{id}\|_{L^{p^{\prime}(\nu) \rightarrow L^{1}(|m|)}} .
$$

Obviously $(3) \Rightarrow(4)$ and $\pi_{1}^{+}\left(\left.I_{m}^{p^{\prime}}\right|_{L^{p^{\prime}}(\nu)}\right)=\left\|T_{m}\right\|$ just taking $T_{m}=I_{m}^{p^{\prime}}$. Therefore it remains the proof of $(4) \Rightarrow(1)$. First note that since

$$
T_{m}\left(\chi_{A}\right)=m(A)=\int_{A} d m=I_{m}^{p^{\prime}}\left(\chi_{A}\right),
$$

for all $A \in \Sigma$, then $T_{m}(s)=I_{m}^{p^{\prime}}(s)$ for all $s \in S_{\Sigma}(\mathbb{R})$ and, by the density of the simple functions in $L^{p^{\prime}}(\nu)$, one gets $T_{m}=I_{m}^{p^{\prime}}$. Take $\pi \in \mathcal{D}_{\Omega}$ and $\sum_{A \in \pi} \alpha_{A} \chi_{A} \in$ $L^{p^{\prime}}(\nu)$. Then

$$
\sum_{A \in \pi}\left|\alpha_{A}\right|\|m(A)\|_{X}=\sum_{A \in \pi}\left\|T_{m}\left(\left|\alpha_{A}\right| \chi_{A}\right)\right\|_{X} \leq \pi_{1}^{+}\left(T_{m}\right)\left\|\sum_{A \in \pi} \alpha_{A} \chi_{A}\right\|_{L^{p^{\prime}(\nu)}} .
$$

This shows that

$$
|m|_{p, \nu} \leq \pi_{1}^{+}\left(T_{m}\right)=\pi_{1}^{+}\left(\left.I_{m}^{p^{\prime}}\right|_{L^{p^{\prime}(\nu)}}\right) .
$$

Similarly to the previous section the vector space consisting of those measures $m$ appearing in Theorem 3.5 is denoted by $\mathrm{V}_{X}^{p}(\nu)$ and we have,

Corollary 3.6. Let $1<p \leq \infty, \nu: \Sigma \rightarrow Y$ a countable additive vector measure and $X$ a Banach space. Then $\mathrm{V}_{X}^{p}(\nu)$ is a Banach space under the norm $|\cdot|_{p, \nu}$ that can be identified isometrically with $\Lambda_{1}^{+}\left(L^{p^{\prime}}(\nu), X\right)$.

Using the results on representation of $p$-convex Banach lattices that we have used in Section 2, we get the integral representation for cone absolutely summing operators.

Corollary 3.7. Let $1<p \leq \infty$. Let $E$ be an order continuous $p^{\prime}$-convex Banach lattice with a weak unit and $X$ a Banach space. Then there is a measure space $(\Omega, \Sigma)$ and a vector measure $\nu$ on it such that $\mathrm{V}_{X}^{p}(\nu)=\Lambda_{1}^{+}(E, X)$ (isomorphically). In particular, there is an order isomorphism $I: E \rightarrow L^{p^{\prime}}(\nu)$ such that for each $T \in \Lambda_{1}^{+}(E, X)$ there is a vector measure $m_{T} \ll \nu$ satisfying that $\left|m_{T}\right|_{p, \nu}<\infty$ and $T(\cdot)=\int I(\cdot) d m_{T}$.

The proof is similar to the one given for Corollary 2.8.

Acknowledgement. J.M. Calabuig and O. Blasco were supported by Ministerio de Economía y Competitividad (Spain) (project MTM2011-23164). E.A. 
Sánchez-Pérez was supported by Ministerio de Economía y Competitividad (Spain) (project MTM2012-36740-C02-02).

\section{REFERENCES}

1. R.C. Bartle, N. Dunford and J.T. Schwartz, Weak compactness and vector measures, Canadian J. Math. 7 (1955), 289-305.

2. O. Blasco, Boundary values of vector-valued harmonic functions considered as operators. Studia Math. 86 (1987), no. 1, 19-33.

3. O. Blasco, Positive p-summing operators, vector measures and tensor products. Proc. Edinburgh Math. Soc. (2) 31 (1988), no. 2, 179-184.

4. O. Blasco, Remarks on the semivariation of vector measures with respect to Banach spaces, Bull. Austral. Math. Soc. 75 (2007), no. 3, 469-480.

5. O. Blasco and J.M. Calabuig, p-variation of vector measures with respect to bilinear maps. Bull. Austral. Math. Soc., 78 (2008), 411-430.

6. J.M. Calabuig, O. Delgado and E.A. Sánchez Pérez, Factorizing operators on Banach function spaces through spaces of multiplication operators, J. Math. Anal. Appl. 364 (2010), 88-103.

7. J. Diestel and J.J. Jr. Uhl, Vector measures. With a foreword by B. J. Pettis. Mathematical Surveys, No. 15. American Mathematical Society, Providence, R.I., 1977. xiii+322 pp.

8. J. Diestel, H. Jarchow and A. Tonge, Absolutely summing operators. Cambridge Studies in Advanced Mathematics, 43. Cambridge University Press, Cambridge, 1995. xvi+474 pp. ISBN: 0-521-43168-9.

9. N. Dinculeanu, Vector measures. International Series of Monographs in Pure and Applied Mathematics, Vol. 95 Pergamon Press, Oxford-New York-Toronto, Ont.; VEB Deutscher Verlag der Wissenschaften, Berlin $1967 \mathrm{x}+432 \mathrm{pp}$.

10. B. Jefferies and S. Okada, Bilinear integration in tensor products. Rocky Mountain J. Math. 28 (1998), no. 2, 517-545.

11. D.R. Lewis, Integration with respect to vector measures, Pacific J. Math. 33(1970), 157-165.

12. D.R. Lewis, On integrability and summability in vector spaces, Illinois J. Math. 16(2) (1972), 294-307.

13. L. Lindenstrauss and L. Tzafriri, Classical Banach Spaces II, Springer, Berlin, 1979.

14. S. Okada, W.J. Ricker and E.A. Sánchez Pérez, Optimal domain and integral extension of operators acting in function spaces, Operator Theory: Advances and Applications, vol. 180, Birkhäuser Verlag, Basel, 2008.

15. E.A. Sánchez Pérez, Spaces of integrable functions with respect to a vector measure of convex range and factorization of operators from Lp-spaces. Pacific J. Math. 207 (2002), 489-495.

16. H.H. Schaefer, Banach lattices and positive operators. Die Grundlehren der mathematischen Wissenschaften, Band 215. Springer-Verlag, New York-Heidelberg, 1974. xi+376 pp.

1 Departamento de Análisis Matemático, Facultad de Matemáticas, Universidad de Valencia, 46100 Burjassot (Valencia), Spain.

E-mail address: oscar.blasco@uv.es

2 Instituto Universitario de Matemática Pura y Aplicada, Universitat Politècnica de València, Camino de Vera s/n, 46022 Valencia, Spain.

E-mail address: jmcalabu@mat.upv.es

2 Instituto Universitario de Matemática Pura y Aplicada, Universitat Politècnica de València, Camino de Vera s/n, 46022 Valencia, Spain.

E-mail address: easancpe@mat.upv.es 